\title{
Reheating in Models with Non-minimal Coupling in metric and Palatini formalisms
}

\author{
Dhong Yeon Cheong, ${ }^{a}$ Sung Mook Lee, ${ }^{a}$ and Seong Chan Park ${ }^{a, b}$ \\ ${ }^{a}$ Department of Physics and IPAP, Yonsei University, Seoul 03722, Republic of Korea \\ ${ }^{b}$ Korea Institute for Advanced Study, Seoul 02455, Republic of Korea \\ E-mail: dhongyeon@yonsei.ac.kr, sungmook.lee@yonsei.ac.kr, \\ sc.park@yonsei.ac.kr
}

\begin{abstract}
We study reheating of inflationary models with general non-minimal coupling $K(\phi) R$ with $K(\phi) \sim \sqrt{V(\phi)}$ where $R$ is the Ricci scalar and $V$ is the inflaton potential. In particular, when we take the monomial potential $K(\phi) \propto \phi^{m}$ with $m \in \mathbb{Z}_{+}$, we provide general analytic expressions for cosmological observables. We consider a wide range of nonminimal coupling $\xi \in[0, \infty)$ in metric and Palatini formalisms and derive the predictions for cosmological observables and the reheating temperature taking a general equation of state parameter $w_{\text {reh }}$.
\end{abstract}




\section{Contents}

1 Introduction 1

2 Model 2

2.1 Non-minimal Coupling in metric and Palatini formalisms 3

2.2 Monomial functions: $K(\phi) \sim \phi^{m} \sim \sqrt{V(\phi)} \quad 4$

$2.3 \xi \ll 1$ (Metric $\approx$ Palatini)

$2.4 \xi \gg 1$ (Metric $\neq$ Palatini) 6

2.4.1 Metric Formalism 7

$\begin{array}{lll}2.4 .2 & \text { Palatini Formalism } & 7\end{array}$

3 Reheating and Cosmological Predictions $\quad 8$

3.1 Reheating 8

3.2 Results : Observables \& Reheating Temperature 9

4 Conclusion and Discussions $\quad 14$

\section{Introduction}

Cosmological inflation [1-3], now widely accepted as a 'standard procedure' in early universe, has been heavily tested with increasing precision [4-6]. However, the particle physics details governing reheating after inflation, which ultimately provides the initial conditions for the hot big bang, is largely unknown. Reheating involves the physics in the perturbative and non-perturbative decay, resonances and nonlinear dynamics of inflaton [7-11].

Phenomenologically, the reheating stage is effectively parameterized by the phenomenological parameters $\left(T_{\text {reh }}, N_{\text {reh }}, w_{\text {reh }}\right)$ where $T_{\text {reh }}$ is the reheating temperature, $N_{\text {reh }}$ is the e-folding number, and $w_{\text {reh }}$ is the (constant) effective equation of state during reheating, respectively. In general, direct cosmological observables are hardly traceable up to the period of reheating, but some indirect bounds are available especially in the single field inflation models: taking the CMB data into account, consistency relations among the parameters $\left(T_{\text {reh }}, N_{\text {reh }}, w_{\text {reh }}\right)$ can be derived $[12,13]$. Numerous studies have been conducted along this line [14-30].

Among the various models compatible with observations that one may consider, models with a non-minimal coupling between the Ricci scalar $R$ and the inflaton field $\phi$ are highly motivated [31-37]. It is known that a generic class of cases exist where the non-minimal coupling $(K(\phi) R)$ induces the asymptotic flatness $\left(\frac{V(\phi)}{K(\phi)^{2}} \sim\right.$ const. $)$, hence guarantees approximate shift symmetry of the inflationary potential and suppresses the tensor-to-scalar ratio $r$ [34, 38]. This class is also known as the $\alpha$-attractor [39]. and it is being constrained by precision CMB measurements (i.e. Planck, WMAP, COBE and BICEP/Keck), and the 
detection of $r$ is one of the main targets of several future experiments including CMB-S4 and LiteBIRD [40,41]. One notable example in the inflationary model class with a non-minimal coupling is the Higgs inflation, where the Standard Model (SM) Higgs $h$ has the role of the inflaton with a non-minimal coupling $K(h)=\xi h^{2}$ and a quartic potential $V(h)=\frac{\lambda}{4} h^{4}$ [42], especially in the vicinity of a critical point [43, 44]. (See Ref. [45] for a recent review.)

Although various theoretical and phenomenological issues of non-minimally coupled inflation models have been explored [31-35, 46-49], in this work, we further refine the cosmological predictions of these models implementing effects of reheating. Reheating is often implicitly assumed to be nearly instantaneous, and conventional wisdom for $\alpha$-attractor class of models (including $R^{2}$-driven Starobinsky model) says the cosmological observables are degenerate among different realizations, but its specific reheating dynamics may depend on microscopic details and alter the observational predictions [50-53]. Considering various theoretical possibilities, we want to cover directions worth pursuing as follows:

- Metric and Palatini formalism: In the Palatini formalism of gravity, the affine connection $\Gamma_{\mu \nu}^{\rho}$ and the metric $g_{\mu \nu}$ are introduced in an independent manner. Although the Palatini formalism is equivalent to the metric formalism at the level of pure Einstein-Hilbert action, it is known that they provide different predictions when non-minimal couplings are present [48, 54-62].

- General monomial potentials: A generic monomial potential $V(\phi) \propto K(\phi)^{2} \propto$ $\phi^{2 m}$ guarantees the asymptotic flat potential in the Einstein frame [34]:

$$
\lim _{\phi \rightarrow \infty}\left(\frac{V}{K^{2}}\right)=\text { Const. }>0 .
$$

The condition essentially describes the $\alpha$-attractor behavior [39]. This criterion is applied in the metric formalism as well as Palatini cases.

- Wide range of non-minimal coupling $\xi \in[0, \infty)$ : Even though large non-minimal couplings are usually considered, a wide range of $\xi$ (including ones much smaller than unity) consistent to observations still remains valid. We consider general cases including $\xi=0$ (minimal, monomial) and $\xi=\infty$ (maximally non-minimal) [12].

This paper is organized as follows. In Section. 2, we set our model in both metric and Palatini formalism of gravity and provide the inflationary predictions. In Section. 3, we relate the cosmological observables $\left(n_{s}, r\right)$ and reheating parameters. We also discuss current/future bound on reheating temperatures from these observables. We conclude in Section. 4.

\section{Model}

In this section, we set the inflationary model with non-minimal coupling term in metric and Palatini formalism. 


\subsection{Non-minimal Coupling in metric and Palatini formalisms}

As the Einstein-Hilbert action already includes the $\left(\right.$ Mass $^{2}{ }^{2}$ dimensional coupling to the Ricci scalar $R$, we are enforced to include the operators of the form $\hat{\mathcal{O}} R$ with $[\hat{\mathcal{O}}]=(\text { Mass })^{2}$ following the effective field theory approach unless a symmetry forbids it. Therefore when we introduce a scalar field $\phi$ to our theory, we include an arbitrary (gauge invariant) function $K(\phi)$ directly coupling to gravity:

$$
S_{J}=\int d^{4} x \sqrt{-g_{J}}\left[-\frac{M^{2}+K(\phi)}{2} R+\frac{1}{2}(\partial \phi)^{2}-V(\phi)\right],
$$

with $[K]=(M a s s)^{2}$ and an arbitrary mass parameter $M$. The potential $V(\phi)$ is a function of $[V]=(M a s s)^{4}$. In metric formalism, the Ricci scalar $R$ is solely determined by the metric $g_{\mu \nu}$, but in the Palatini formalism it is also determined by the connection $\Gamma_{\nu \lambda}^{\mu}$ which is taken to be independent of the metric. In the present of non-minimal coupling $K(\phi) R$, the two formalisms are not equivalent and predicts different Universe [54].

By performing the Weyl transformation,

$$
g_{E, \mu \nu}=\Omega^{2} g_{J, \mu \nu}, \quad \quad \Omega^{2} \equiv \frac{M^{2}+K(\phi)}{M_{P}^{2}}
$$

the action is conveniently transformed into the canonical form of gravity in Einstein frame

$$
S=\int d^{4} x \sqrt{-g_{E}}\left[-\frac{M_{P}^{2}}{2} R_{E}+\frac{1}{2} \Pi(\phi)(\partial \phi)^{2}-\frac{V(\phi)}{\Omega^{4}}\right]
$$

where the non-trivial kinetic term is given by

$$
\Pi(\phi) \equiv \frac{1}{\Omega^{2}}+\frac{3 \zeta}{2 M_{P}^{2}} \frac{K^{\prime}(\phi)^{2}}{\Omega^{4}}, \quad \zeta=\left\{\begin{array}{ll}
1 & \text { (Metric) } \\
0 & \text { (Palatini) }
\end{array} .\right.
$$

in metric and Palatini formalism, respectively. The second term $\left(\propto K^{\prime 2}\right)$ originates from the transformation of the Ricci scalar $R(\Gamma)$, making it absent in the Palatini formalism. The kinetic term is easily canonicalized by $\sqrt{\Pi(\phi)} \partial h=\partial \phi$ or

$$
\frac{d h}{d \phi}=\sqrt{\Pi(\phi)}
$$

When the explicit function $K(\phi)$ is given, we obtain the canonical scalar $h(\phi)$ by integration.

An asymptotically flat, positive potential in the Einstein frame $V_{E}(h(\phi))$ should satisfy a general condition at large fields [34]:

$$
\lim _{\phi \rightarrow \infty} \frac{V(\phi)}{K(\phi)^{2}}=\text { Const. }>0
$$

We request this condition $V \sim K^{2}$ or equivalently $K \sim \sqrt{V}$ to realize successful slowroll inflation. We emphasize again that the condition essentially describes the $\alpha$-attractor behavior [39]. 
2.2 Monomial functions: $K(\phi) \sim \phi^{m} \sim \sqrt{V(\phi)}$

To explicitly see the inflationary behavior, we choose $K$ and $V$ to be monomial functions with $m \geq 1^{1}$

$$
K(\phi)=\xi M_{P}^{2}\left(\frac{\phi}{M_{P}}\right)^{m}, \quad V=\frac{\lambda M_{P}^{4}}{2 m}\left(\frac{\phi}{M_{P}}\right)^{2 m},
$$

where $\xi$ and $\lambda$ are arbitrary dimensionless parameters. In addition, without a (or with a sufficiently small) vacuum expectation value of the field $\phi$, we have $M \simeq M_{P}$ to guarantee the canonicalized Einstein frame field (later we define as $h$ ) and potential $U(\phi(h))$ coincide with the Jordan frame field $\phi$ and potential $V(\phi)$ near the origin. Therefore, we set $M=M_{P}$ without losing precision in our predictions. Then the potential in Einstein frame becomes

$$
U(\phi)=\frac{M_{P}^{4}}{\left(M_{P}^{2}+K\right)^{2}} V=\frac{\lambda M_{P}^{4}\left(\frac{\phi}{M_{P}}\right)^{2 m}}{2 m\left(1+\xi\left(\frac{\phi}{M_{P}}\right)^{m}\right)^{2}} .
$$

The slow-roll parameters are defined in the Einstein frame:

$$
\begin{aligned}
& \epsilon(\phi) \equiv \frac{M_{P}^{2}}{2}\left(\frac{\partial U(\phi(h)) / \partial h}{U}\right)^{2}=\frac{M_{P}^{2}}{2 \Pi_{\zeta}(\phi)}\left(\frac{\partial U / \partial \phi}{U}\right)^{2} \\
& \eta(\phi) \equiv M_{P}^{2} \frac{\partial^{2} U / \partial h^{2}}{U}=\frac{M_{P}^{2}}{\sqrt{\Pi_{\zeta}(\phi)} U} \frac{\partial}{\partial \phi}\left(\frac{1}{\sqrt{\Pi_{\zeta}(\phi)}} \frac{\partial U}{\partial \phi}\right),
\end{aligned}
$$

where $\Pi_{\zeta}$ is defined in Eq. (2.4) for metric $(\zeta=1)$ and Palatini $(\zeta=0)$ cases with $K(\phi)$ in Eq. (2.7) and $x=\phi / M_{P}$ :

$$
\Pi_{\zeta}\left(\phi=M_{P} x\right)=\frac{1+\xi x^{m}+\zeta \frac{3 m^{2} \xi^{2}}{2} x^{2(m-1)}}{\left(1+\xi x^{m}\right)^{2}}, \quad \zeta=\left\{\begin{array}{ll}
1 & \text { (Metric) } \\
0 & \text { (Palatini) }
\end{array} .\right.
$$

The explicit form of the slow-roll parameters are obtained

$$
\begin{aligned}
\epsilon(\phi & \left.=M_{P} x\right)=\frac{4 m^{2}}{2 x^{2}\left(\xi x^{m}+1\right)+3 \zeta m^{2} \xi^{2} x^{2 m}}, \\
\eta\left(\phi=M_{P} x\right) & =\frac{4 m\left[x^{2}\left(\xi x^{m}+1\right)\left\{(m+2) \xi x^{m}-4 m+2\right\}-3 \zeta m^{3} \xi^{2} x^{2 m}\left(\xi x^{m}-1\right)\right]}{\left[2 x^{2}\left(\xi x^{m}+1\right)+3 \zeta m^{2} \xi^{2} x^{2 m}\right]^{2}} .
\end{aligned}
$$

It is noticed that the slow-roll parameters are independent of $\lambda$.

The cosmological time (in Einstein frame) $t_{k}$ when the mode corresponding to the pivot scale $k$ leaves the horizon is determined by $k=a\left(t_{k}\right) H\left(t_{k}\right)$ with the scale factor $a$ and $H$ being the Hubble parameter. In this work, the pivot scale is chosen to be $k=0.05 \mathrm{Mpc}^{-1}$. On the other hand, the time $t_{e}$ at the end of the inflation is set by $\epsilon\left(\phi\left(t_{e}\right)\right)=1$.

\footnotetext{
${ }^{1}$ For models with a global $U(1)$ symmetry, a $K(\phi)$ function with an odd power of $\phi$ is usually forbidden.
} 
Then, the number of e-foldings during expansion from a pivot scale with $a_{k}=a\left(t_{k}\right)$ to the end of inflation at $a_{e}=a\left(t_{e}\right)$ is given by

$$
\begin{aligned}
N\left(\phi_{k}\right) \equiv N_{k} & =\log \frac{a_{e}}{a_{k}}=\frac{1}{M_{P}^{2}} \int_{h\left(\phi_{e}\right)}^{h\left(\phi_{k}\right)} d h \frac{U}{\partial U / \partial h} \\
& =\int_{\phi_{e}}^{\phi_{k}} \frac{d \phi}{M_{P}} \sqrt{\frac{\Pi_{\zeta}(\phi)}{2 \epsilon(\phi)}}=\left[\frac{x^{2}}{4 m}+\frac{3}{4} \zeta\left(\xi x^{m}-\ln \left(M_{P}^{m}+\zeta M_{P}^{m} x^{m}\right)\right)\right]_{\phi_{e} / M_{P}}^{\phi_{k} / M_{P}}
\end{aligned}
$$

with $\phi_{k} \equiv \phi\left(t_{k}\right), \phi_{e} \equiv \phi\left(t_{e}\right)$, and $H_{k} \equiv H\left(t_{k}\right)$ where we used $d h=d \phi \sqrt{\Pi_{\zeta}}$ in the 2nd line.

\section{$2.3 \xi \ll 1($ Metric $\approx$ Palatini $)$}

We first analyze the $\xi \ll 1$ limit close to the minimal case. The exact slow-roll parameters Eq. (2.12) and Eq. (2.13) in this limit then take the approximate expression

$$
\begin{aligned}
& \epsilon\left(\phi=M_{P} x\right) \simeq \frac{2 m^{2}}{x^{2}}-2 m^{2} \xi x^{m-2}+\left(2 x^{2}-3 m^{2} \zeta\right) m^{2} \xi^{2} x^{2 m-4}+\mathcal{O}\left(\xi^{3}\right) \\
& \eta\left(\phi=M_{P} x\right) \simeq \frac{2 m(2 m-1)}{x^{2}}-5 m^{2} \xi x^{m-2}+\left[5 x^{2}+3(2-3 m) m \zeta\right] m^{2} \xi^{2} x^{2 m-4}+\mathcal{O}\left(\xi^{3}\right)
\end{aligned}
$$

where $\zeta=0,1$ each correspond to the Palatini and metric cases, respectively. Note that the $\zeta$ parameter dependence appears in the $\mathcal{O}\left(\xi^{2}\right)$ order, i.e. the predictions of both cases will deviate in $\mathcal{O}\left(\xi^{2}\right)$, and the observables approximately coincide for $\xi \ll 1$.

The field value where inflation ends for both cases is determined by $\epsilon=1$, hence

$$
\phi_{e} \simeq \sqrt{2} m M_{P}-2^{\frac{m-1}{2}} m^{1+m} M_{P} \xi+2^{m-\frac{5}{2}} m^{2 m+1}(2 m+3-3 \zeta) M_{P} \xi^{2}+\mathcal{O}\left(\xi^{3}\right) .
$$

Consequently, the e-folding number $N_{k}$ from the pivot scale $a_{k}$ to the end of inflation $a_{e}$ then becomes, following Eq. (2.14)

$$
N_{k} \simeq \frac{\phi_{k}^{2}-\phi_{e}^{2}}{4 m M_{P}^{2}}+\frac{3}{8} \zeta\left[\frac{\phi_{k}^{2 m}-\phi_{e}^{2 m}}{M_{P}^{2 m}}\right] \xi^{2}
$$

As $\xi \ll 1$, the field value $\phi_{k}$ at pivot scale is represented in terms of $N_{k}$ as

$$
\phi_{k} \simeq \sqrt{2 m\left(m+2 N_{k}\right)} M_{P}-\frac{2^{\frac{m}{2}-\frac{1}{2}} m^{m+2}}{\sqrt{m\left(m+2 N_{k}\right)}} M_{P} \xi+\mathcal{O}\left(\xi^{2}\right)
$$

leading to the slow-roll parameters

$$
\begin{aligned}
\epsilon\left(\phi_{k}\right) & \simeq \frac{m}{m+2 N_{k}}+\frac{2^{\frac{m}{2}}\left[m^{m+2}-m^{\frac{m}{2}+1}\left(m+2 N_{k}\right)^{\frac{m}{2}+1}\right] \xi}{\left(m+2 N_{k}\right)^{2}}+\mathcal{O}\left(\xi^{2}\right), \\
\eta\left(\phi_{k}\right) & \simeq \frac{2 m-1}{2 N_{k}+m}+\frac{2^{\frac{m}{2}-1}\left[2 m^{m+1}(2 m-1)-5 m^{\frac{m}{2}+1}\left(m+2 N_{k}\right)^{\frac{m}{2}+1}\right] \xi}{\left(m+2 N_{k}\right)^{2}}+\mathcal{O}\left(\xi^{2}\right) .
\end{aligned}
$$


These expressions yield the spectral index $n_{s}$ and the tensor-to-scalar ratio $r$ in this limit as

$$
\begin{aligned}
n_{s} & \simeq 1-6 \epsilon+\left.2 \eta\right|_{\phi=\phi_{k}} \\
& \simeq 1-\frac{2(m+1)}{m+2 N_{k}}+\frac{2^{\frac{m}{2}}\left[m^{\frac{m}{2}+1}\left(m+2 N_{k}\right)^{\frac{m}{2}+1}-2 m^{m+1}(m+1)\right] \xi}{\left(m+2 N_{k}\right)^{2}}+\mathcal{O}\left(\xi^{2}\right) \\
r & \left.\simeq 16 \epsilon\right|_{\phi=\phi_{k}} \simeq \frac{16 m}{m+2 N_{k}}+\frac{2^{\frac{m}{2}+4}\left[m^{m+2}-m^{\frac{m}{2}+1}\left(m+2 N_{k}\right)^{\frac{m}{2}+1}\right] \xi}{\left(m+2 N_{k}\right)^{2}}+\mathcal{O}\left(\xi^{2}\right)
\end{aligned}
$$

along with the curvature power spectrum amplitude being

$$
\begin{aligned}
A_{s} \simeq & \left.\frac{1}{24 \pi^{2} \epsilon} \frac{U}{M_{P}^{4}}\right|_{\phi=\phi_{k}} \\
\simeq & \frac{2^{m-4} m^{m-2}\left(m+2 N_{k}\right)^{m+1} \lambda}{3 \pi^{2}} \\
& \quad-\frac{2^{\frac{3 m}{2}-4} m^{\frac{3 m}{2}-2} \lambda\left[(m+1) m^{\frac{m}{2}+1}\left(m+2 N_{k}\right)^{m}+\left(m+2 N_{k}\right)^{\frac{3 m}{2}+1}\right] \xi}{3 \pi^{2}}
\end{aligned}
$$

where $A_{s} \simeq 2.1 \times 10^{-9}$ is given by Planck and BICEP/Keck 2018 results at the pivot scale $k=0.05 \mathrm{Mpc}^{-1}[5]$. The $\zeta$ dependent terms are suppressed, with its leading order terms emerging at $\mathcal{O}\left(\xi^{2}\right)$ order.

Note that, the specific $\xi$ range that resembles an approximate minimal coupling case differs for each $m$ value. In order for a noticeable deviation of $\left(n_{s}, r\right)$ from minimal cases, the required $\delta \xi$ involves

$$
\left|\frac{\delta r}{r}\right| \sim\left|\frac{\delta n_{s}}{n_{s}}\right| \sim\left(4 m N_{k}\right)^{\frac{m}{2}}|\delta \xi|
$$

which is $m$ dependent. A larger power monomial potential will lead to a smaller $\delta \xi$ that shows a reasonable deviation from $\xi=0$ predictions.

In the minimal coupling case $(\xi=0)$, the relations of the inflationary observables simplify to

$$
n_{s} \simeq 1-\frac{m+1}{N_{k}}, \quad r \simeq \frac{8 m}{N_{k}},
$$

and these two parameters are correlated as

$$
\left.N_{k} \simeq \frac{m+1}{1-n_{s}} \simeq \frac{8 m}{r} \quad \Leftrightarrow \quad r \simeq \frac{8 m}{m+1}\left(1-n_{s}\right) \approx \frac{0.32 m}{m+1}\right|_{n_{s} \approx 0.96} .
$$

The resulting value of $r$ is a bit too large compared to the observed limit $r \lesssim 0.1[4-6]$.

\section{$2.4 \xi \gg 1$ (Metric $\neq$ Palatini)}

We now turn to the opposite limit and examine the case with a large non-vanishing nonminimal coupling. Now the predictions from metric and Palatini formalisms drastically differ. Therefore we discuss the metric case and Palatini case below separately. 


\subsubsection{Metric Formalism}

In the large $\xi$ limit, slow roll parameters are approximated as

$$
\begin{aligned}
\epsilon & \simeq \frac{4}{3 \xi^{2}}\left(\frac{M_{P}}{\phi}\right)^{2 m}+\mathcal{O}\left(\xi^{-3}\right), \\
\eta & \simeq-\frac{4}{3 \xi}\left(\frac{M_{P}}{\phi}\right)^{m}+\frac{4}{3 \xi^{2}}\left[\left(\frac{M_{P}}{\phi}\right)^{2 m}+\frac{3 m-2}{3 m^{3}}\left(\frac{M_{P}}{\phi}\right)^{2 m-2}\right]+\mathcal{O}\left(\xi^{-3}\right) .
\end{aligned}
$$

Therefore, $\epsilon\left(\phi_{e}\right)=1$ gives

$$
\phi_{e} \simeq\left(\frac{4}{3 \xi^{2}}\right)^{\frac{1}{2 m}} M_{P} .
$$

E-folding number and the field value at the pivot scale are obtained

$$
N_{k} \approx \frac{3 \xi}{4}\left(\frac{\phi_{k}}{M_{P}}\right)^{m} \quad \Rightarrow \quad \phi_{k} \simeq\left(\frac{4 N_{k}}{3 \xi}\right)^{\frac{1}{m}} M_{P}, \quad(\xi \gg 1, \text { Metric }) .
$$

Then, the slow roll parameters and cosmological observables are represented by $N_{k}$ as

$$
\epsilon\left(\phi_{k}\right) \simeq \frac{3}{4 N_{k}^{2}}, \quad \eta\left(\phi_{k}\right) \simeq-\frac{1}{N_{k}}+\frac{3}{4 N_{k}^{2}}+\frac{3 m-2}{4 N_{k}^{2} m^{3}}\left(\frac{4 N_{k}}{3 \xi}\right)^{\frac{2}{m}}
$$

and the spectral index and tensor-to-scalar-ratio are

$$
n_{s} \simeq 1-6 \epsilon+\left.2 \eta\right|_{\phi=\phi_{k}} \simeq 1-\frac{2}{N_{k}}-\frac{3}{N_{k}^{2}},\left.\quad r_{s} \simeq 16 \epsilon\right|_{\phi=\phi_{k}} \simeq \frac{12}{N_{k}^{2}},
$$

which enjoys the sweet spot of the Planck, WMAP and BICEP/Keck bounds on $\left(n_{s}, r\right)$ for $N_{k} \simeq 50-60$. Prospects from future measurements are discussed in Section. 3.

From the fact that the potential during inflation approaches a constant value at the large field regime with a large non-minimal coupling,

$$
U(\phi) \stackrel{\xi \gg 1, \phi \gg M_{P} / \xi^{1 / m}}{\longrightarrow} U_{\mathrm{inf}} \simeq \frac{\lambda M_{P}^{4}}{2 m \xi^{2}}
$$

we have the normalization of $\lambda / \xi^{2}$ as

$$
\left.A_{s} \simeq \frac{1}{24 \pi^{2} \epsilon} \frac{U}{M_{P}^{4}}\right|_{\phi=\phi_{k} \gg M_{P} / \xi^{1 / m}} \simeq \frac{N_{k}^{2} \lambda}{36 m \pi^{2} \xi^{2}} \simeq 2.1 \times 10^{-9} .
$$

\subsubsection{Palatini Formalism}

The slow-roll parameters in Palatini formalism are approximated as

$$
\epsilon \simeq \frac{2 m^{2} M_{P}^{m+2}}{\xi \phi^{m+2}}, \quad \eta \simeq-\frac{m(m+2) M_{P}^{2}}{\phi^{2}} .
$$

By requiring $\epsilon\left(\phi_{e}\right)=1$,

$$
\phi_{e}=2^{\frac{1}{2+m}} M_{P}\left(\frac{\xi}{m^{2}}\right)^{-\frac{1}{2+m}} .
$$


The e-folding number and the field value at pivot scale are

$$
N_{k} \simeq \frac{1}{4 m M^{2}}\left(\phi_{k}^{2}-\phi_{e}^{2}\right) \quad \Rightarrow \quad \phi_{k} \simeq 2 \sqrt{m N_{k}} M_{P}, \quad(\xi \gg 1 \text {, Palatini })
$$

where we neglect $\phi_{e} \ll \phi_{k}$. Now slow roll parameters and cosmological observables are represented as

$$
\epsilon\left(\phi_{k}\right) \simeq \frac{m^{1-\frac{m}{2}}}{2^{1+m} N_{k}^{1+\frac{m}{2}} \xi}, \quad \eta\left(\phi_{k}\right) \simeq-\frac{2+m}{4 N_{k}}+\frac{5 m^{1-\frac{m}{2}}}{2^{2+m} N_{k}^{1+\frac{m}{2}} \xi}
$$

and the spectral index and the tensor-to-scalar ratio are

$$
n_{s} \simeq 1-\frac{2+m}{2 N_{k}}-\frac{m^{1-\frac{m}{2}}}{2^{1+m} N_{k}^{1+\frac{m}{2}} \xi}, \quad r \simeq \frac{m^{1-\frac{m}{2}}}{2^{m-3} N_{k}^{1+\frac{m}{2}} \xi} .
$$

One should note that $r \propto 1 / \xi$ is highly suppressed by $\xi \gg 1$. In Palatini case, CMB power spectrum normalization determines $\lambda / \xi$ as

$$
\left.A_{s} \simeq \frac{1}{24 \pi^{2} \epsilon} \frac{U}{M_{P}^{4}}\right|_{\phi \simeq \phi_{k}} \simeq \frac{2^{m-3} m^{\frac{m}{2}-2} N_{k}^{\frac{m}{2}+1} \lambda}{3 \pi^{2} \xi} .
$$

The fact that $\lambda / \xi$ is normalized requires a larger order of magnitude of $\xi$ for the same $\lambda$ compared to metric cases.

\section{Reheating and Cosmological Predictions}

\subsection{Reheating}

In this section we enlist the calculations of $N_{\text {reh }}$ and $T_{\text {reh }}$ by modeling the reheating epoch to be described with a constant equation of state $w_{\text {reh }}$. Note that the equation of state $w(t) \equiv p / \rho$, in general, is a time dependent parameter determined by the particle physics details of the reheating process [63-66]. In our approach following Ref. [12], we rather take $w_{\text {reh }}$ as an average value over the reheating

$$
w_{\mathrm{reh}} \equiv \frac{1}{N_{\mathrm{reh}}} \int_{N_{k}}^{N_{k}+N_{\mathrm{reh}}} w(N) d N
$$

In this work, we allows a wide range of $w_{\text {reh }} \in[0,1 / 3]$. Recall that $w=0$ for matter domination and $w=1 / 3$ for radiation domination. ${ }^{2}$

Now we take the standard assumptions for the late time cosmology after the reheating and get the relations for $T_{\text {reh }}$ and $N_{\text {reh }}$. First, the e-folds for the epoch is expressed as

$$
N_{\mathrm{reh}} \equiv \ln \left(\frac{a_{\mathrm{reh}}}{a_{e}}\right)=\frac{1}{3\left(1+w_{\mathrm{reh}}\right)} \ln \left(\frac{\rho_{e}}{\rho_{\mathrm{reh}}}\right),
$$

where $N_{\text {reh }}$ represents the duration of reheating from the end of inflation, $a_{\text {reh }}$ and $a_{e}$ is the scale factor at the end of reheating and the end of inflation, respectively, while $\rho_{\text {reh }}$ and $\rho_{e}$

\footnotetext{
${ }^{2}$ In principle, one might take $w_{\mathrm{reh}} \in[-1,1]$ depending on the detail of the model.
} 
is the corresponding energy density at the end of reheating and the end of inflation. From $\epsilon_{H}=-\frac{\dot{H}}{H^{2}}=1$ at the end of inflation, we have

$$
\rho_{e}=\frac{3}{2} U_{e}
$$

hence $w\left(t_{e}\right)=-1 / 3$. Also, the radiation energy with the relativistic degrees of freedom $g_{\text {reh }}$ and the temperature $T_{\text {reh }}$ at the end of reheating is given by

$$
\rho_{\mathrm{reh}}=\frac{\pi^{2}}{30} g_{\mathrm{reh}} T_{\mathrm{reh}}^{4}
$$

Assuming no additional entropy production after reheating, we can link $T_{\text {reh }}$ and the temperature of our current universe $T_{0}$ as

$$
T_{\mathrm{reh}}=T_{0}\left(\frac{a_{0}}{a_{\mathrm{reh}}}\right)\left(\frac{g_{0}}{g_{\mathrm{reh}}}\right)^{1 / 3}=\left(\frac{43}{11 g_{\mathrm{reh}}}\right)^{1 / 3}\left(\frac{a_{0} T_{0}}{k}\right) H_{k} e^{-N_{k}} e^{-N_{\mathrm{reh}}}
$$

where $g_{0}=2+\frac{7}{8} \times N_{\text {eff }} \times \frac{4}{11}$ with $N_{\text {eff }}=3.046$ in the $\mathrm{SM}$ is the relativistic degree of freedom at current universe and $g_{\mathrm{reh}}$ is that at the end of reheating, respectively. In the second line, we take the length of e-folds of radiation dominance, $N_{\mathrm{RD}}=\ln \left(a_{\mathrm{eq}} / a_{\text {reh }}\right)$ with the scale factor at the matter-radiation equality $a_{\mathrm{eq}}$, and the ratio $a_{0} / a_{\mathrm{eq}}=a_{0}\left(H_{k} / k\right) e^{-N_{k}} e^{-N_{\mathrm{reh}}} e^{-N_{\mathrm{RD}}}$ from the pivot scale $k=a_{k} H_{k}$.

Combining the expressions, we obtain $N_{\text {reh }}$ as

$$
\begin{aligned}
N_{\mathrm{reh}}=\frac{4}{3\left(1+w_{\mathrm{reh}}\right)}\left[\frac{1}{4} \ln \left(\frac{45}{\pi^{2} g_{\mathrm{reh}}}\right)+\ln \left(\frac{U_{e}^{1 / 4}}{H_{k}}\right)\right. & +\frac{1}{3} \ln \left(\frac{11 g_{\mathrm{reh}}}{43}\right) \\
+ & \left.\ln \left(\frac{k}{a_{0} T_{0}}\right)+N_{k}+N_{\mathrm{reh}}\right] .
\end{aligned}
$$

Taking the standard values $g_{\text {reh }}=106.75, k=0.05 \mathrm{Mpc}^{-1}, T_{0}=2.725 \mathrm{~K}$ for $w_{\text {reh }} \neq-1 / 3$ we arrive at

$$
N_{\text {reh }}=\frac{4}{\left(1-3 w_{\text {reh }}\right)}\left[61.6-\ln \left(\frac{U_{e}^{1 / 4}}{H_{k}}\right)-N_{k}\right],
$$

and accordingly, inserting this expression to $T_{\text {reh }}$ gives

$$
T_{\mathrm{reh}}=\left[\left(\frac{43}{11 g_{\mathrm{reh}}}\right)^{1 / 3} \frac{a_{0} T_{0}}{k} H_{k} e^{-N_{k}}\left(\frac{45 U_{e}}{\pi^{2} g_{\mathrm{reh}}}\right)^{-\frac{1}{3\left(1+w_{\mathrm{reh}}\right)}}\right]^{\frac{3\left(1+w_{\mathrm{reh}}\right)}{3 w_{\mathrm{reh}}-1}} .
$$

\subsection{Results : Observables \& Reheating Temperature}

We present the exact results in Figure. 1 for metric cases and Figure. 2, Figure. 3 for Palatini cases. For all figures, we include Planck18+BICEP/Keck(BK)18+BAO $1 \sigma$ (yellow) and $2 \sigma$ (green) results on $\left(n_{s}, r\right)$ as well as prospective results from future CMB-S4 observations with a fiducial detection of $r=3 \times 10^{-3}$ (purple) and null results (blue), both assuming a similar $n_{s}$ center value with Planck18+BK18+BAO [6, 40]. 
To begin with, we discuss how inflationary predictions differ in common in both metric and Palatini formalisms. First, the predictions of $\left(n_{s}, r\right)$ change depending on $\left(w_{\text {reh }}, T_{\text {reh }}\right)$. For illustration, we denote predictions on $\left(n_{s}, r\right)$ for $w_{\text {reh }}=0$ (black) and $w_{\text {reh }}=1 / 5$ (brown) depending on $T_{\text {reh }} \in\left[10^{-2} \mathrm{GeV}-T_{\max }\right]$. $T_{\max }$ is determined by imposing instantaneous reheating

$$
\rho_{e}=\frac{3}{2} U_{e}=\frac{\pi^{2}}{30} g_{\mathrm{reh}} T_{\max }^{4}
$$

at the end of the inflation. Lines corresponding to $T_{\max }$ are denoted by solid lines. For metric cases, $T_{\max } \simeq 10^{16} \mathrm{GeV}$ and for Palatini cases, $T_{\max }$ decreases for larger $\xi$. (See Figure. 6.) On the other hand, the lower bound $T_{\text {reh }} \sim 10^{-2} \mathrm{GeV}$ corresponds to the BBN scale temperature $T_{\mathrm{BBN}}$ as a conservative assumption and is depicted in dotted lines. Dot-dashed and dashed lines correspond to $T_{\text {reh }}=10^{5} \mathrm{GeV}$ and $T_{\text {reh }}=10^{10} \mathrm{GeV}$, respectively. ${ }^{3}$ Lower reheating temperature gives smaller $n_{s}$ predictions while the $r$ dependence on reheating temperature is weak. Constraints on the reheating temperature from current/future $n_{s}$ and $r$ observations are also discussed below.

Second, the regions of prediction become narrower and converge to the solid line on the right as $w_{\text {reh }} \rightarrow 1 / 3$. In this limit, the end of the reheating is ambiguous, because there is no distinct change in the equation of state compared to the radiation dominant phase. Instead, the predictions on $\left(n_{s}, r\right)$ does not depend on reheating processes and is uniquely determined solely by inflationary model parameters.

We note that, depending on $m$, even $\xi \lesssim \mathcal{O}(1)$ still provides predictions compatible to current constraints. For example, in the metric formalism, intermediate $\xi$ values $\xi \sim \mathcal{O}(1)$ for $m=1$, and $\xi \sim \mathcal{O}\left(10^{-1}\right)$ for $m=2$ and each of these show different predictions for $\left(n_{s}, r\right)$, but still fully consistent to observations.

On the other hand, there are several notable differences between the two formalisms of gravity. For the Palatini case, each $m$ give different predictions even in the $\xi \rightarrow \infty$ limit. Potentials with the form $m \geq 3$ for $w_{\text {reh }} \in\left[0, \frac{1}{3}\right]$ are ruled out regardless of the reheating history. This is shown in Figure. 2. (However, if one considers $w_{\mathrm{reh}}>1 / 3$, the predictions shift to larger $n_{s}$ values so that these can be inside current observational bounds.)

Also, in the Palatini case, from the fact that $\left.r\right|_{\xi \rightarrow \infty} \propto 1 / \xi$, the tensor-to-scalar ratio is highly suppressed, while for metric cases, predictions converge to specific values depending on $T_{\text {reh }}$ as long as $\xi \gg 1$. This was precisely why, Palatini Higgs inflation has hardly been regarded to be proven by observation in general. However, in this work, we show that the predictions of $n_{s}$ for non-minimal inflation in the Palatini formalism actually possesses a dependence on $\xi$, when we take the reheating processes more carefully.

In the $m=1$ case, the predictions are still highly compatible with the CMB observations, regardless of the $w_{\text {reh }}$ value. Interestingly, for $m=2$ in the Palatini case, we observe that quartic potential inflation with a large non-minimal coupling $\xi \gtrsim \mathcal{O}(10)$ is already outside of current Planck18+BK18+BAO $1 \sigma$ bounds, even for instantaneous reheating. (See

\footnotetext{
${ }^{3} T_{\text {reh }}=10^{5} \mathrm{GeV}$ can be understood as an energy scale of future collider experiments for new physics searches. $T_{\text {reh }}=10^{10} \mathrm{GeV}$ is usually regarded as a typical upper bound of the reheating temperature of the 'gravitino overproduction problem' if one takes this literally.
} 

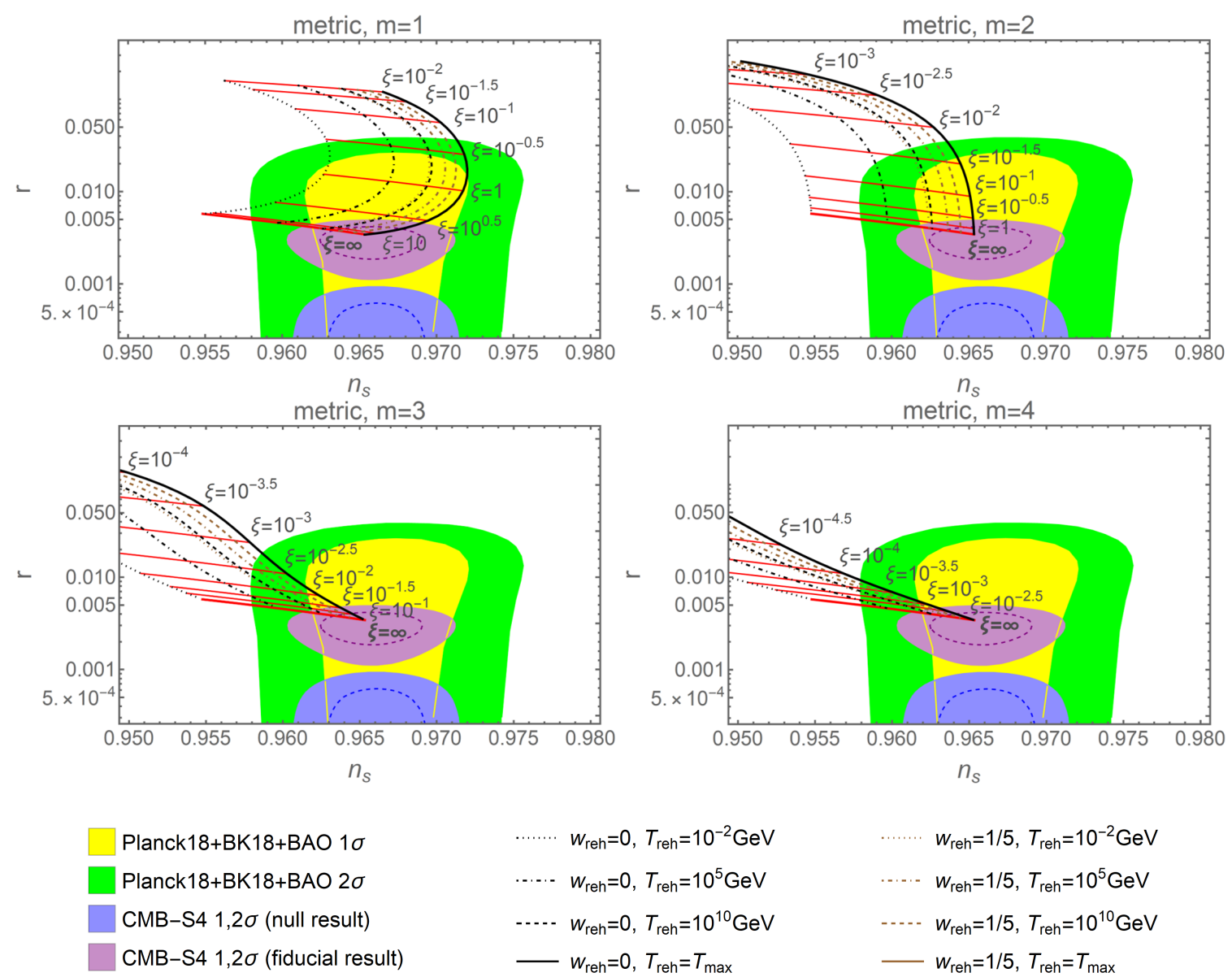

$$
\begin{aligned}
& \cdots \cdots w_{\text {reh }}=0, T_{\text {reh }}=10^{-2} \mathrm{GeV} \\
& \cdots \cdots w_{\text {reh }}=0, T_{\text {reh }}=10^{5} \mathrm{GeV} \\
& \cdots w_{\text {reh }}=0, T_{\text {reh }}=10^{10} \mathrm{GeV} \\
& -w_{\text {reh }}=0, T_{\text {reh }}=T_{\text {max }}
\end{aligned}
$$$$
\text { …..... } w_{\text {reh }}=1 / 5, T_{\text {reh }}=10^{-2} \mathrm{GeV}
$$$$
\text { …. } W_{\text {reh }}=1 / 5, T_{\text {reh }}=10^{5} \mathrm{GeV}
$$$$
\text { -... } W_{\text {reh }}=1 / 5, T_{\text {reh }}=10^{10} \mathrm{GeV}
$$$$
w_{\text {reh }}=1 / 5, T_{\text {reh }}=T_{\max }
$$

Figure 1. $\left(n_{s}, r\right)$ plot with $m=(1,2,3,4)$ in metric formalism. Red solid lines shows the dependence of inflationary predictions on various $\xi \geq 0$ values for $w_{\text {reh }} \in\left[0, \frac{1}{3}\right]$ and $T_{\text {reh }} \in\left[T_{\mathrm{BBN}}, T_{\mathrm{max}}\right]$. Dotted, dash-dotted, dashed, solid lines denote inflationary predictions with $T_{\text {reh }}=\left(10^{-2}, 10^{5}, 10^{10}, T_{\max }\right) \mathrm{GeV}$ respectively, along with $w_{\text {reh }}=0$ for black and $w_{\text {reh }}=\frac{1}{5}$ for brown lines. Current Planck18+BK18+BAO and expected future CMB-S4 measurements are denoted each in yellow/green and blue/purple.

the right of Figure. 3.) This also includes conventionally considered non-minimal coupling values for Palatini-Higgs inflation with $\xi \gtrsim \mathcal{O}\left(10^{8}\right)$ (See Eq. (2.43) with $\lambda \gtrsim \mathcal{O}\left(10^{-2}\right)$ ). Also, $w_{\text {reh }}$ values deviating from $\frac{1}{3}$ tend to worsen the compatibility with observations for lower $T_{\text {reh }}$. For example, for $\xi=10^{8}$, current CMB bounds require $T_{\text {reh }} \gtrsim \mathcal{O}\left(10^{10}\right) \mathrm{GeV}$ for $w_{\text {reh }}=0$ and $T_{\text {reh }} \gtrsim \mathcal{O}\left(10^{5}\right) \mathrm{GeV}$ for $w_{\text {reh }}=\frac{1}{5}$. These constraints will be more stringent for future observations such as CMB-S4 [40] and LiteBIRD [41], lying beyond the $2 \sigma$ expected bounds. This fact also can be used to distinguish metric Higgs inflation models from Palatini cases [48, 67-72]. Note that the early stage of the reheating (namely 'preheating') of Palatini Higgs inflation possesses $w \simeq-1$ [73], which makes the compatibility to the observations worse if the reheating processes has an averaged equation of state $w_{\text {reh }}<1 / 3 .{ }^{4}$

\footnotetext{
${ }^{4}$ As the reheating stage of the Palatini Higgs inflation is tachyonic, we expect the reheating to be very
} 




Figure 2. $\left(n_{s}, r\right)$ plot for $m=(1,2,3,4)$ in Palatini formalism. Solid lines are for instantaneous reheating, and dotted lines are for $T_{\text {reh }}=T_{\mathrm{BBN}}$. Except for $m=1$ and $m=2$, other power potentials are inconsistent to current observations, regardless of the reheating temperature for equation of state $w_{\text {reh }} \in\left[0, \frac{1}{3}\right]$.
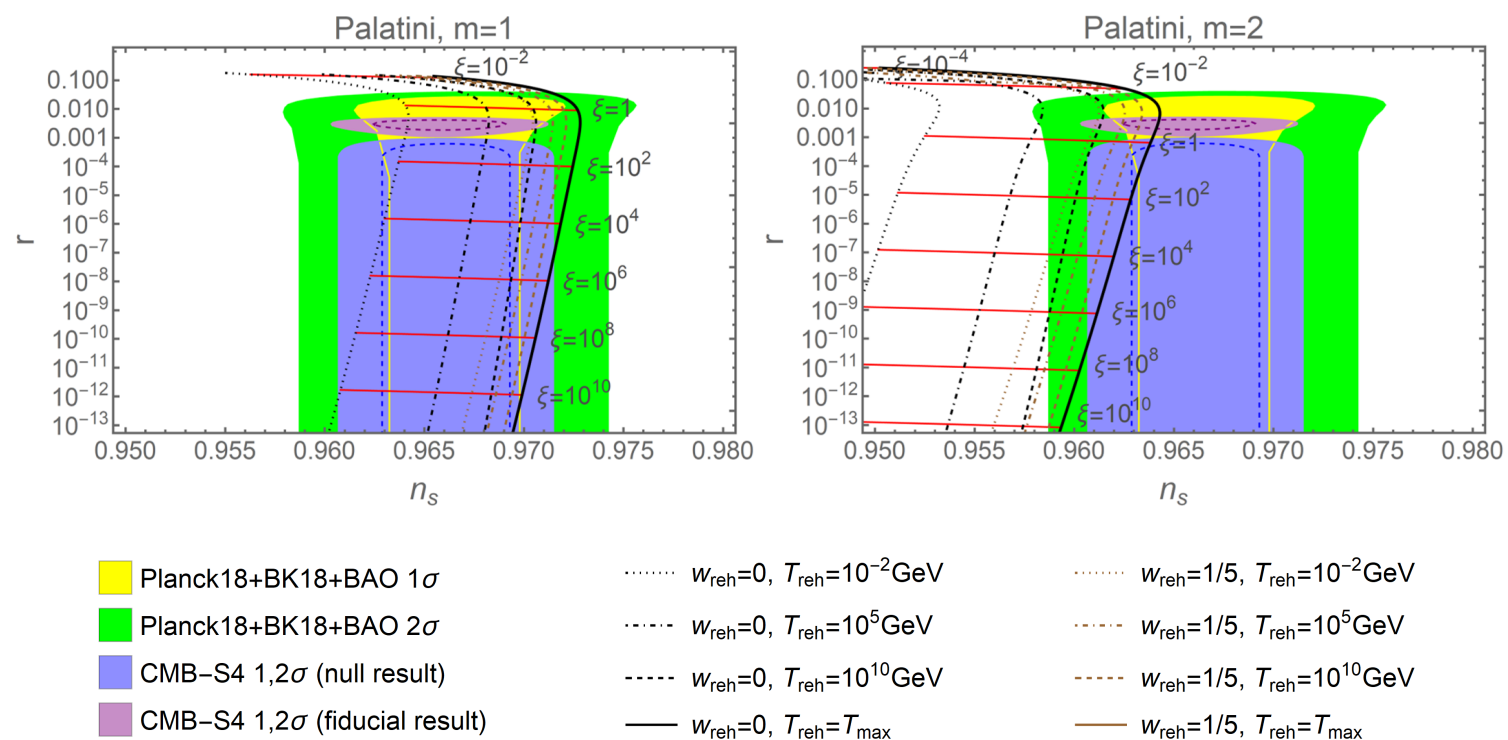

$$
\begin{aligned}
& \cdots \cdots w_{\text {reh }}=0, T_{\text {reh }}=10^{-2} \mathrm{GeV} \\
& \cdots \cdots w_{\text {reh }}=0, T_{\text {reh }}=10^{5} \mathrm{GeV} \\
& \cdots w_{\text {reh }}=0, T_{\text {reh }}=10^{10} \mathrm{GeV} \\
& -w_{\text {reh }}=0, T_{\text {reh }}=T_{\text {max }}
\end{aligned}
$$$$
\text { …..... } W_{\text {reh }}=1 / 5, T_{\text {reh }}=10^{-2} \mathrm{GeV}
$$$$
\begin{aligned}
& \cdots w_{\text {reh }}=1 / 5, T_{\text {reh }}=10^{5} \mathrm{GeV} \\
& \cdots W_{\text {reh }}=1 / 5, T_{\text {reh }}=10^{10} \mathrm{GeV} \\
& -w_{\text {reh }}=1 / 5, T_{\text {reh }}=T_{\max }
\end{aligned}
$$

Figure 3. $\left(n_{s}, r\right)$ plot for $m=1$ and $m=2$ in Palatini formalism. Plotting conventions are the same as Figure. 1. Depending on the reheating temperature, predictions on $n_{s}$ significantly changes as well.

Following the preceding results, bounds on $\left(n_{s}, r\right)$ also impose constraints on $T_{\text {reh }}$ depending on $w_{\text {reh }}$. Relations between $\left(n_{s}, T_{\text {reh }}\right)$ for metric cases are depicted in Figure. 4.

efficient so that $w_{\text {reh }}$ is very close to $1 / 3$. However, detailed analysis on the reheating of this model and determination of the precise $w_{\text {reh }}$ is out of the scope of this work. 


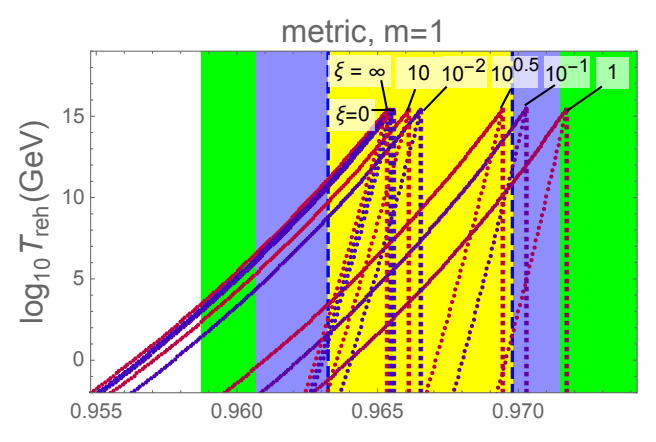

$n_{S}$

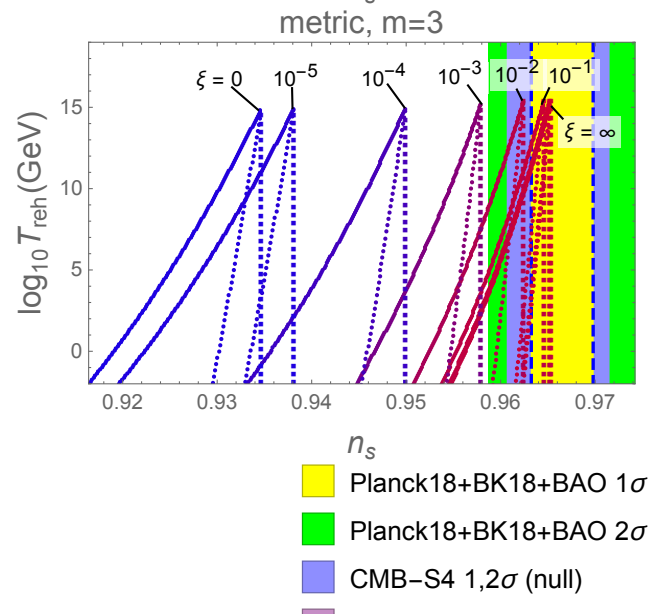

CMB-S4 $1,2 \sigma$ (fiducial $r=0.003)$



Figure 4. Constraints on the reheating temperature $T_{\text {reh }}$ depending on $n_{s}$ for the metric $m=$ $(1,2,3,4)$ cases. The spectrum of $r$ is also depicted. The solid, point-dotted, square-dotted lines each correspond to $w_{\text {reh }}=0, w_{\text {reh }}=1 / 5, w_{\text {reh }}=1 / 3$, respectively. The Planck $18+\mathrm{BK} 18+\mathrm{BAO} 1 \sigma$ and $2 \sigma$ constraints are depicted in yellow and green, respectively. Future CMB-S4 null and fiducial constraints are also expressed in blue and purple, where the dashed lines and solid boundaries represent $1 \sigma$ and $2 \sigma$ bounds.

The $\xi$ values range from $\xi=0$ to $\xi=\infty$ for all $m=(1,2,3,4)$ potentials. The allowed range of $T_{\text {reh }}$ varies depending on the potential form along with the specific non-minimal coupling value. We also note that, for $m=1$, there exists some range of $\xi$ which gives upper bound on $T_{\text {reh }}$ as well depending on $w_{\text {reh }}$. The $\left(r, T_{\text {reh }}\right)$ relation for metric cases is also shown in Figure. 5. As the prospected sensitivity at CMB-S4 improves significantly compared to Planck18+BK18+BAO up to $r \lesssim 10^{-3}$, these observations may be used to effectively constrain $T_{\text {reh }}$.

The $T_{\text {reh }}$ implications for the Palatini case with $m=(1,2)$, which is depicted in Figure. 6, differ from the metric scenario. The suppression of $r \propto 1 / \xi$ in the Palatini formalism makes it nearly impossible for future observations to provide reasonable constraints on the allowed $T_{\text {reh }}$ range for large $\xi$ parameters. Instead, the $\xi$ dependence on $n_{s}$ may lead to providing a more stringent (upper/lower) bound on $T_{\text {reh }}$. 

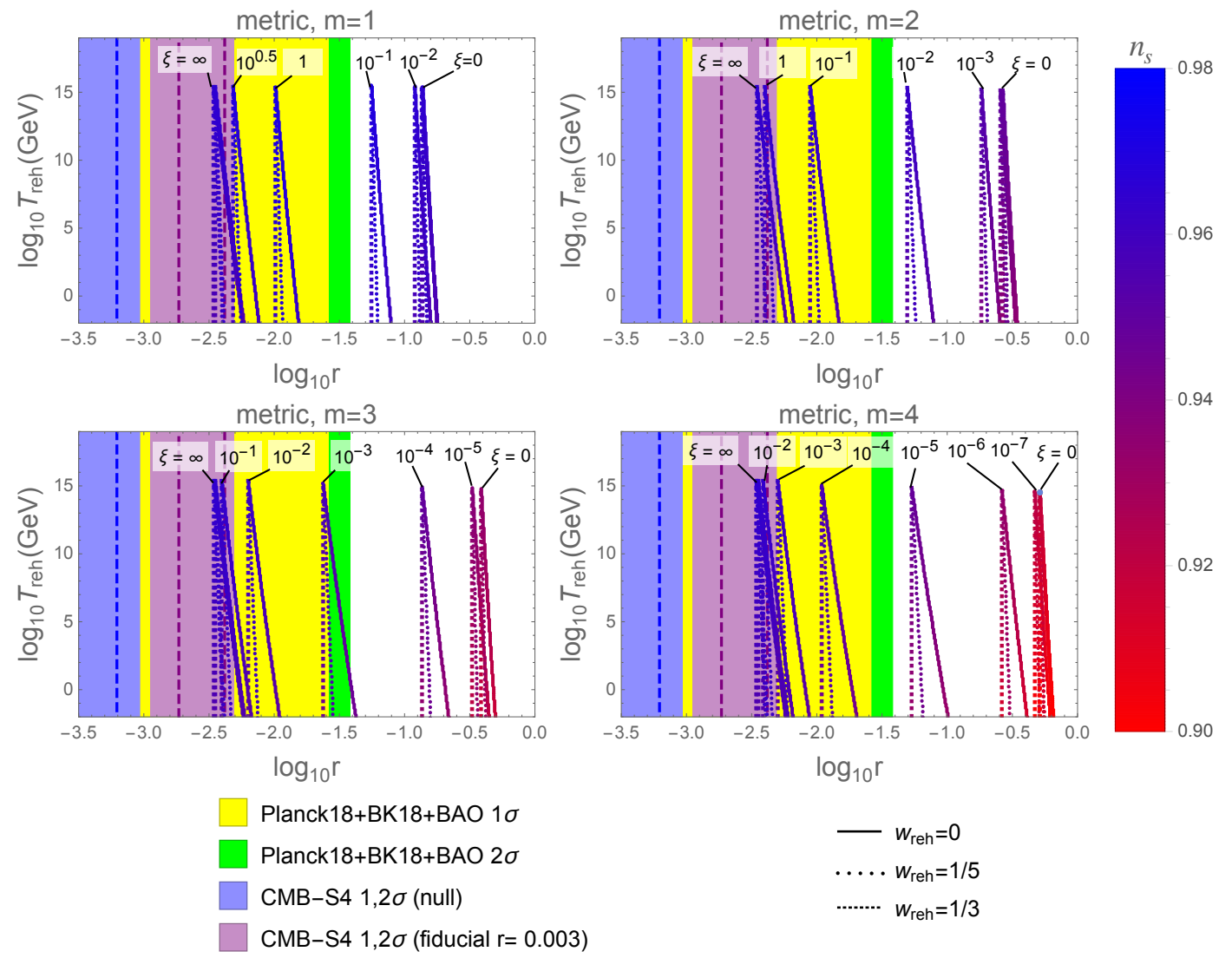

Figure 5. Constraints on the reheating temperature $T_{\text {reh }}$ depending on $r$ for the metric $m=$ $(1,2,3,4)$ cases. The spectrum of $n_{s}$ is also depicted. The solid, point-dotted, square-dotted lines each correspond to $w_{\text {reh }}=0, w_{\text {reh }}=1 / 5, w_{\text {reh }}=1 / 3$, respectively. The Planck $18+\mathrm{BK} 18+\mathrm{BAO} 1 \sigma$ and $2 \sigma$ constraints are depicted in yellow and green, respectively. Future CMB-S4 null and fiducial constraints are also expressed in blue and purple, where the dashed lines and solid boundaries represent $1 \sigma$ and $2 \sigma$ bounds.

\section{Conclusion and Discussions}

In this work, we clarified the effects of reheating to predictions of inflation models with non-minimal coupling to gravity, covering (i) both metric and Palatini formalisms, (ii) arbitrary monomial potential with asymptotic flatness in the Einstein frame, and (iii) a wide range of non-minimal coupling $\xi$ depending on the power of the potential, from the consistency relations between reheating parameters and inflationary observables $n_{s}$ and $r$. We also obtained a range of compatible reheating temperatures for a given equation of state parameter $w_{\text {reh }}$ during reheating. This in turn can be used complementarily to further decipher the microscopic particle physics governing the reheating process for each model.

For models in both formalisms, we note that the CMB compatible $\xi$ values also allow $\xi \leq 1$, with its predictions deviating from those for large $\xi \gg 1$ limits. The specific dependence on $\left(n_{s}, r\right)$ in this particular $\xi$ range differ with a definite dependence on the details of the model's potential. 

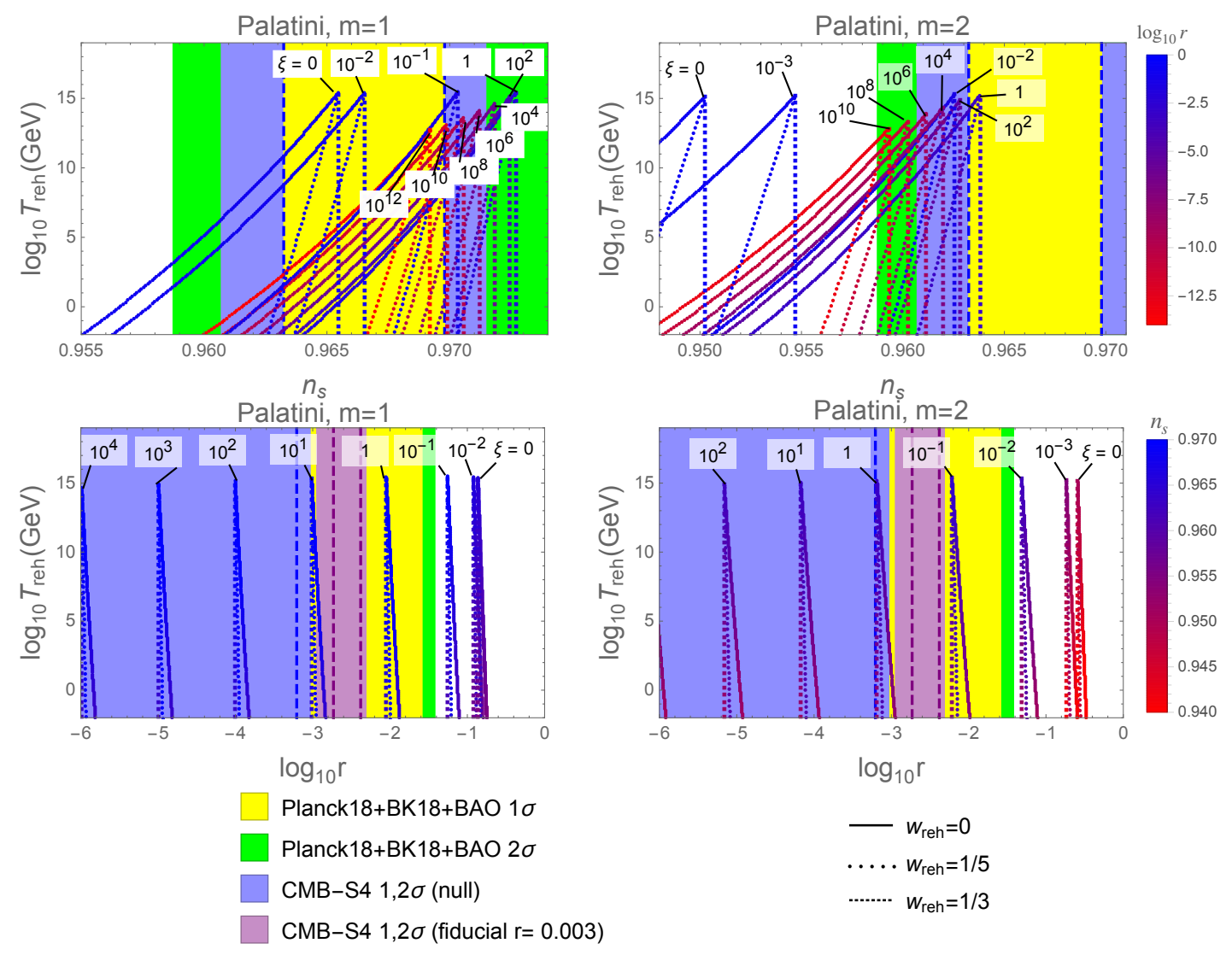

Figure 6. Constraints on the reheating temperature $T_{\text {reh }}$ depending on $n_{s}$ (top) and $r$ (bottom) for the Palatini $m=1$ and $m=2$ cases. The spectrum of $r$ and $n_{s}$ is also depicted accordingly. The solid, point-dotted, square-dotted lines each correspond to $w_{\text {reh }}=0, w_{\text {reh }}=1 / 5, w_{\text {reh }}=1 / 3$, respectively. The Planck18+BK18+BAO $1 \sigma$ and $2 \sigma$ constraints are depicted in yellow and green, respectively. Future CMB-S4 null and fiducial constraints are also expressed in blue and purple, where the dashed lines and solid boundaries represent $1 \sigma$ and $2 \sigma$ bounds. For cases $m=3,4$, the predictions contradict with current CMB observations in terms of $n_{s}$, regardless of the reheating dynamics.

Especially, for $m=2$ in the Palatini formalism, while $\xi \gtrsim \mathcal{O}\left(10^{-2}\right)$ are allowed for current CMB $2 \sigma$ bounds, the dependence on $\xi$ indicates that typically considered large $\xi \sim \mathcal{O}\left(10^{8}\right)$ lead to $N_{k}$ smaller than 50 , which is not preferred within the $1 \sigma$ limit of current CMB bounds. Current and future CMB observations are able to constrain the particular parameter range of $\xi \mathrm{s}$, giving definite lower and upper bounds on the non-minimal coupling, with the constraints being stronger when taking the effects of reheating into account.

We emphasize that our results are applicable to a wider class of models, which exhibit $\alpha$-attractor behavior. $R^{2}$-driven Starobinsky inflation is also transformable to this class, giving the same inflationary dynamics. Application to multi-field inflationary models that are reducible into an effective single-field $\alpha$-attractor case is also straightforward. However, the reheating details of these equivalent inflationary classes differ depending specifically on the model that describes its microscopic nature, and predicts different values of 
$\left(T_{\text {reh }}, N_{\text {reh }}, w_{\text {reh }}\right)$. Therefore, our result could be used as a generic template for the inflationary predictions of wide classes of models, breaking the degeneracy of the various models not caught solely from considering the inflation dynamics. This approach will provide valuable information on deciphering the contents of the post-inflationary universe, which may feature - but most definitely not restricted to - multiple fields encompassing spectator fields, new interactions including higher order gravitational couplings. ${ }^{5}$ We will further investigate this direction in future works.

\section{Acknowledgments}

We thank Fedor Bezrukov, Kin-ya Oda, and Minxi He for discussions throughout various stages of the research. This work is supported in part by the National Research Foundation of Korea (NRF) grant funded by the Korea government (NRF-2019R1A2C1089334), (NRF2021R1A4A2001897) (SCP), and (NRF-2020R1A6A3A13076216) (SML). SML is supported by the Hyundai Motor Chung Mong-Koo Foundation Scholarship.

\section{References}

[1] A. H. Guth, "The Inflationary Universe: A Possible Solution to the Horizon and Flatness Problems," Phys. Rev. D 23 (1981) 347-356.

[2] A. D. Linde, "A New Inflationary Universe Scenario: A Possible Solution of the Horizon, Flatness, Homogeneity, Isotropy and Primordial Monopole Problems," Phys. Lett. B 108 (1982) 389-393.

[3] A. Albrecht and P. J. Steinhardt, "Cosmology for Grand Unified Theories with Radiatively Induced Symmetry Breaking," Phys. Rev. Lett. 48 (1982) 1220-1223.

[4] WMAP Collaboration, E. Komatsu et al., "Seven-Year Wilkinson Microwave Anisotropy Probe (WMAP) Observations: Cosmological Interpretation," Astrophys. J. Suppl. 192 (2011) 18, arXiv:1001.4538 [astro-ph.CO].

[5] Planck Collaboration, Y. Akrami et al., "Planck 2018 results. X. Constraints on inflation," Astron. Astrophys. 641 (2020) A10, arXiv:1807.06211 [astro-ph.C0].

[6] BICEP/Keck Collaboration, P. A. R. Ade et al., "Improved Constraints on Primordial Gravitational Waves using Planck, WMAP, and BICEP/Keck Observations through the 2018 Observing Season," Phys. Rev. Lett. 127 no. 15, (2021) 151301, arXiv: 2110.00483 [astro-ph.CO].

[7] A. D. Dolgov and D. P. Kirilova, "ON PARTICLE CREATION BY A TIME DEPENDENT SCALAR FIELD," Sov. J. Nucl. Phys. 51 (1990) 172-177.

[8] J. H. Traschen and R. H. Brandenberger, "Particle Production During Out-of-equilibrium Phase Transitions," Phys. Rev. D 42 (1990) 2491-2504.

[9] L. Kofman, A. D. Linde, and A. A. Starobinsky, "Reheating after inflation," Phys. Rev. Lett. 73 (1994) 3195-3198, arXiv:hep-th/9405187.

[10] Y. Shtanov, J. H. Traschen, and R. H. Brandenberger, "Universe reheating after inflation," Phys. Rev. D 51 (1995) 5438-5455, arXiv:hep-ph/9407247.

\footnotetext{
${ }^{5}$ Single field inflation models including higher order $R^{3}$ have been analyzed in Ref. [74].
} 
[11] L. Kofman, A. D. Linde, and A. A. Starobinsky, "Towards the theory of reheating after inflation," Phys. Rev. D 56 (1997) 3258-3295, arXiv:hep-ph/9704452.

[12] J. L. Cook, E. Dimastrogiovanni, D. A. Easson, and L. M. Krauss, "Reheating predictions in single field inflation," JCAP 04 (2015) 047, arXiv:1502.04673 [astro-ph.CO].

[13] M. Drewes, "What can the CMB tell about the microphysics of cosmic reheating?," JCAP 03 (2016) 013, arXiv:1511.03280 [astro-ph.CO].

[14] R.-G. Cai, Z.-K. Guo, and S.-J. Wang, "Reheating phase diagram for single-field slow-roll inflationary models," Phys. Rev. D 92 (2015) 063506, arXiv:1501.07743 [gr-qc].

[15] T. Rehagen and G. B. Gelmini, "Low reheating temperatures in monomial and binomial inflationary potentials," JCAP 06 (2015) 039, arXiv: 1504.03768 [hep-ph] .

[16] J. Ellis, M. A. G. Garcia, D. V. Nanopoulos, and K. A. Olive, "Calculations of Inflaton Decays and Reheating: with Applications to No-Scale Inflation Models," JCAP 07 (2015) 050, arXiv: 1505.06986 [hep-ph].

[17] R. C. de Freitas and S. V. B. Gonçalves, "CMB Constraints on Reheating Models with Varying Equation of State," arXiv:1509.08500 [astro-ph.C0].

[18] Y. Ueno and K. Yamamoto, "Constraints on $\alpha$-attractor inflation and reheating," Phys. Rev. D 93 no. 8, (2016) 083524, arXiv:1602.07427 [astro-ph.Co].

[19] I. Dalianis, G. Koutsoumbas, K. Ntrekis, and E. Papantonopoulos, "Reheating predictions in Gravity Theories with Derivative Coupling," JCAP 02 (2017) 027, arXiv: 1608.04543 [gr-qc].

[20] M. Drewes, J. U. Kang, and U. R. Mun, "CMB constraints on the inflaton couplings and reheating temperature in $\alpha$-attractor inflation," JHEP 11 (2017) 072, arXiv:1708.01197 [astro-ph.CO].

[21] T. Krajewski, K. Turzyński, and M. Wieczorek, "On preheating in $\alpha$-attractor models of inflation," Eur. Phys. J. C 79 no. 8, (2019) 654, arXiv:1801.01786 [astro-ph.C0].

[22] N. Rashidi and K. Nozari, " $\alpha$-Attractor and reheating in a model with noncanonical scalar fields," Int. J. Mod. Phys. D 27 no. 07, (2018) 1850076, arXiv:1802.09185 [astro-ph.C0].

[23] A. Di Marco, G. Pradisi, and P. Cabella, "Inflationary scale, reheating scale, and pre-BBN cosmology with scalar fields," Phys. Rev. D 98 no. 12, (2018) 123511, arXiv:1807.05916 [astro-ph.co].

[24] Z. Sakhi, A. Safsafi, M. Ferricha-Alami, H. Chakir, and M. Bennai, "Observational constraints on reheating in braneworld inflation," Int. J. Mod. Phys. A 34 no. 27, (2019) 1950152.

[25] I. D. Gialamas and A. B. Lahanas, "Reheating in $R^{2}$ Palatini inflationary models," Phys. Rev. D 101 no. 8, (2020) 084007, arXiv:1911.11513 [gr-qc].

[26] K. Asadi and K. Nozari, "Reheating constraints on a two-field inflationary model," Nucl. Phys. B 949 (2019) 114827.

[27] G. German, "Model independent results for the inflationary epoch and the breaking of the degeneracy of models of inflation," JCAP 11 (2020) 006, arXiv:2003.09420 [astro-ph.CO].

[28] N. Das and S. Panda, "Inflation and Reheating in $\mathrm{f}(\mathrm{R}, \mathrm{h})$ theory formulated in the Palatini formalism," JCAP 05 (2021) 019, arXiv:2005.14054 [gr-qc]. 
[29] J. Haro and L. Aresté Saló, "Note on the reheating temperature in Starobinsky-type potentials," Gen. Rel. Grav. 52 no. 12, (2020) 116, arXiv:2005.14653 [gr-qc].

[30] A. D. Kanfon, F. Mavoa, and S. M. J. Houndjo, " $\alpha$-attractor and reheating in exponential harmonic field model," Astrophys. Space Sci. 365 no. 6, (2020) 97.

[31] T. Futamase and K.-i. Maeda, "Chaotic Inflationary Scenario in Models Having Nonminimal Coupling With Curvature," Phys. Rev. D 39 (1989) 399-404.

[32] R. Fakir and W. G. Unruh, "Improvement on cosmological chaotic inflation through nonminimal coupling," Phys. Rev. D 41 (1990) 1783-1791.

[33] E. Komatsu and T. Futamase, "Complete constraints on a nonminimally coupled chaotic inflationary scenario from the cosmic microwave background," Phys. Rev. D 59 (1999) 064029, arXiv:astro-ph/9901127.

[34] S. C. Park and S. Yamaguchi, "Inflation by non-minimal coupling," JCAP 08 (2008) 009, arXiv:0801.1722 [hep-ph].

[35] M. P. Hertzberg, "On Inflation with Non-minimal Coupling," JHEP 11 (2010) 023, arXiv:1002.2995 [hep-ph].

[36] J. Kim, Y. Kim, and S. C. Park, "Two-field inflation with non-minimal coupling," Class. Quant. Grav. 31 (2014) 135004, arXiv:1301.5472 [hep-ph].

[37] S. C. Park and C. S. Shin, "Clockwork inflation with non-minimal coupling," Eur. Phys. J. C 79 no. 6, (2019) 529, arXiv:1807.09952 [hep-ph].

[38] S. C. Park, "A Class of inflation models with non-minimal coupling," J. Korean Phys. Soc. 55 (2009) 2136-2139, arXiv:0809.4536 [hep-ph].

[39] R. Kallosh, A. Linde, and D. Roest, "Superconformal Inflationary $\alpha$-Attractors," JHEP 11 (2013) 198, arXiv:1311.0472 [hep-th].

[40] K. Abazajian et al., "CMB-S4 Science Case, Reference Design, and Project Plan," arXiv: 1907.04473 [astro-ph.IM].

[41] LiteBIRD Collaboration, M. Hazumi et al., "LiteBIRD: JAXA's new strategic L-class mission for all-sky surveys of cosmic microwave background polarization," Proc. SPIE Int. Soc. Opt. Eng. 11443 (2020) 114432F, arXiv:2101.12449 [astro-ph.IM].

[42] F. L. Bezrukov and M. Shaposhnikov, "The Standard Model Higgs boson as the inflaton," Phys. Lett. B 659 (2008) 703-706, arXiv:0710.3755 [hep-th].

[43] Y. Hamada, H. Kawai, K.-y. Oda, and S. C. Park, "Higgs inflation from Standard Model criticality," Phys. Rev. D 91 (2015) 053008, arXiv:1408.4864 [hep-ph].

[44] Y. Hamada, H. Kawai, K.-y. Oda, and S. C. Park, "Higgs Inflation is Still Alive after the Results from BICEP2," Phys. Rev. Lett. 112 no. 24, (2014) 241301, arXiv:1403. 5043 [hep-ph].

[45] D. Y. Cheong, S. M. Lee, and S. C. Park, "Progress in Higgs inflation," J. Korean Phys. Soc. 78 no. 10, (2021) 897-906, arXiv:2103.00177 [hep-ph].

[46] N. Makino and M. Sasaki, "The Density perturbation in the chaotic inflation with nonminimal coupling," Prog. Theor. Phys. 86 (1991) 103-118.

[47] A. Linde, M. Noorbala, and A. Westphal, "Observational consequences of chaotic inflation with nonminimal coupling to gravity," JCAP 03 (2011) 013, arXiv:1101.2652 [hep-th]. 
[48] R. Jinno, M. Kubota, K.-y. Oda, and S. C. Park, "Higgs inflation in metric and Palatini formalisms: Required suppression of higher dimensional operators," JCAP 03 (2020) 063, arXiv:1904.05699 [hep-ph].

[49] S. M. Lee, K.-y. Oda, and S. C. Park, "Spontaneous Leptogenesis in Higgs Inflation," JHEP 03 (2021) 083, arXiv:2010.07563 [hep-ph].

[50] D. J. H. Chung, E. W. Kolb, and A. Riotto, "Production of massive particles during reheating," Phys. Rev. D 60 (1999) 063504, arXiv:hep-ph/9809453.

[51] G. F. Giudice, E. W. Kolb, and A. Riotto, "Largest temperature of the radiation era and its cosmological implications," Phys. Rev. D 64 (2001) 023508, arXiv:hep-ph/0005123.

[52] S. Davidson and S. Sarkar, "Thermalization after inflation," JHEP 11 (2000) 012, arXiv:hep-ph/0009078.

[53] K. Harigaya and K. Mukaida, "Thermalization after/during Reheating," JHEP 05 (2014) 006, arXiv:1312.3097 [hep-ph].

[54] F. Bauer and D. A. Demir, "Inflation with Non-Minimal Coupling: Metric versus Palatini Formulations," Phys. Lett. B 665 (2008) 222-226, arXiv:0803.2664 [hep-ph].

[55] N. Tamanini and C. R. Contaldi, "Inflationary Perturbations in Palatini Generalised Gravity," Phys. Rev. D 83 (2011) 044018, arXiv:1010.0689 [gr-qc].

[56] A. Borowiec, M. Kamionka, A. Kurek, and M. Szydlowski, "Cosmic acceleration from modified gravity with Palatini formalism," JCAP 02 (2012) 027, arXiv:1109.3420 [gr-qc].

[57] T. Tenkanen, "Resurrecting Quadratic Inflation with a non-minimal coupling to gravity," JCAP 12 (2017) 001, arXiv: 1710.02758 [astro-ph.CO].

[58] A. Racioppi, "Coleman-Weinberg linear inflation: metric vs. Palatini formulation," JCAP 12 (2017) 041, arXiv:1710.04853 [astro-ph.CO].

[59] L. Järv, A. Racioppi, and T. Tenkanen, "Palatini side of inflationary attractors," Phys. Rev. D 97 no. 8, (2018) 083513, arXiv:1712.08471 [gr-qc].

[60] A. Racioppi, "New universal attractor in nonminimally coupled gravity: Linear inflation," Phys. Rev. D 97 no. 12, (2018) 123514, arXiv:1801.08810 [astro-ph.CO].

[61] T. Tenkanen, "Tracing the high energy theory of gravity: an introduction to Palatini inflation," Gen. Rel. Grav. 52 no. 4, (2020) 33, arXiv:2001.10135 [astro-ph.CO].

[62] L. Järv, A. Karam, A. Kozak, A. Lykkas, A. Racioppi, and M. Saal, "Equivalence of inflationary models between the metric and Palatini formulation of scalar-tensor theories," Phys. Rev. D 102 no. 4, (2020) 044029, arXiv:2005.14571 [gr-qc].

[63] D. I. Podolsky, G. N. Felder, L. Kofman, and M. Peloso, "Equation of state and beginning of thermalization after preheating," Phys. Rev. D 73 (2006) 023501, arXiv:hep-ph/0507096.

[64] K. D. Lozanov and M. A. Amin, "Equation of State and Duration to Radiation Domination after Inflation," Phys. Rev. Lett. 119 no. 6, (2017) 061301, arXiv:1608.01213 [astro-ph.CO].

[65] P. Saha, S. Anand, and L. Sriramkumar, "Accounting for the time evolution of the equation of state parameter during reheating," Phys. Rev. D 102 no. 10, (2020) 103511, arXiv:2005.01874 [astro-ph.CO].

[66] A. Di Marco and G. Pradisi, "Variable Inflaton Equation of State and Reheating," arXiv:2102.00326 [gr-qc]. 
[67] F. Bauer and D. A. Demir, "Higgs-Palatini Inflation and Unitarity," Phys. Lett. B 698 (2011) 425-429, arXiv: 1012.2900 [hep-ph].

[68] S. Rasanen and P. Wahlman, "Higgs inflation with loop corrections in the Palatini formulation," JCAP 11 (2017) 047, arXiv:1709.07853 [astro-ph.CO].

[69] T. Markkanen, T. Tenkanen, V. Vaskonen, and H. Veermäe, "Quantum corrections to quartic inflation with a non-minimal coupling: metric vs. Palatini," JCAP 03 (2018) 029, arXiv:1712.04874 [gr-qc].

[70] M. Shaposhnikov, A. Shkerin, and S. Zell, "Quantum Effects in Palatini Higgs Inflation," JCAP 07 (2020) 064, arXiv:2002.07105 [hep-ph].

[71] I. D. Gialamas, A. Karam, A. Lykkas, and T. D. Pappas, "Palatini-Higgs inflation with nonminimal derivative coupling," Phys. Rev. D 102 no. 6, (2020) 063522, arXiv:2008.06371 [gr-qc].

[72] V.-M. Enckell, S. Nurmi, S. Räsänen, and E. Tomberg, "Critical point Higgs inflation in the Palatini formulation," JHEP 04 (2021) 059, arXiv:2012.03660 [astro-ph.CO].

[73] J. Rubio and E. S. Tomberg, "Preheating in Palatini Higgs inflation," JCAP 04 (2019) 021, arXiv: 1902.10148 [hep-ph].

[74] D. Y. Cheong, H. M. Lee, and S. C. Park, "Beyond the Starobinsky model for inflation," Phys. Lett. B 805 (2020) 135453, arXiv:2002.07981 [hep-ph]. 